Engineer Research and

Development Center

Army Terrestrial Environmental Modeling and Intelligence System (ARTEMIS)

\title{
Image Analysis and Classification Based on Soil Strength
}

Ariana M. Sopher, Sally A. Shoop, Jesse M. Stanley, Jr., and

Brian T. Tracy

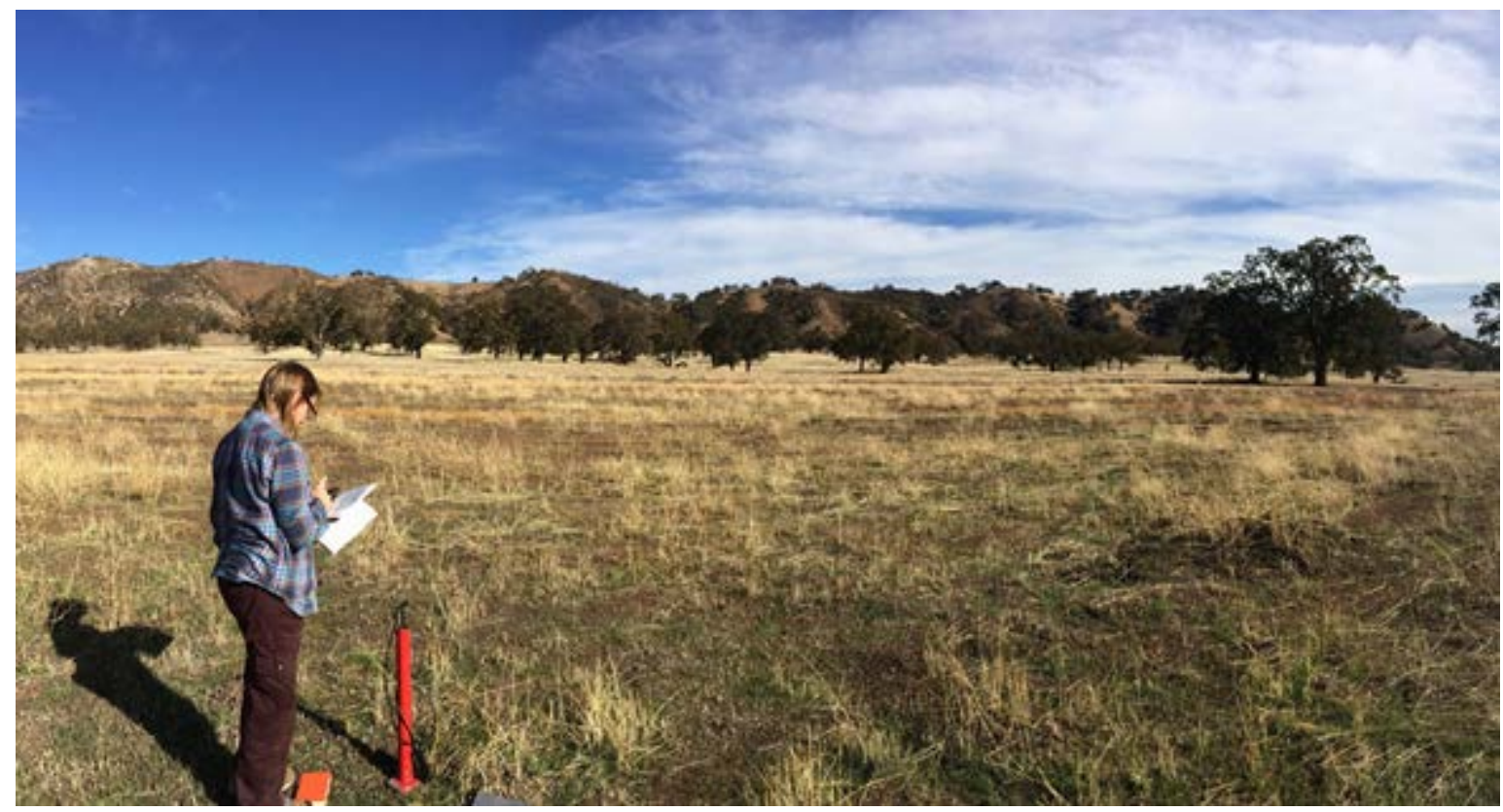


The U.S. Army Engineer Research and Development Center (ERDC) solves the nation's toughest engineering and environmental challenges. ERDC develops innovative solutions in civil and military engineering, geospatial sciences, water resources, and environmental sciences for the Army, the Department of Defense, civilian agencies, and our nation's public good. Find out more at www.erdc.usace.army.mil.

To search for other technical reports published by ERDC, visit the ERDC online library at http://acwc.sdp.sirsi.net/client/default. 


\section{Image Analysis and Classification Based on Soil Strength}

Ariana M. Sopher, Sally A. Shoop, Jesse M. Stanley, Jr., and Brian T. Tracy

U.S. Army Engineer Research and Development Center (ERDC)

Cold Regions Research and Engineering Laboratory (CRREL)

72 Lyme Road

Hanover, NH 03755-1290

Final Report

Approved for public release; distribution is unlimited.

Prepared for Army Terrestrial Environmental Modeling and Intelligence System (ARTEMIS)

U.S. Army Engineer Research and Development Center (ERDC)

Cold Regions Research and Engineering Laboratory (CRREL)

72 Lyme Road

Hanover, NH 03755-1290

Under Work Item 6CJ4D3 for the Geospatial Remote Assessment for Ingress

Locations (GRAIL) Project 


\section{Abstract}

Satellite imagery classification is useful for a variety of commonly used applications, such as land use classification, agriculture, wetland delineation, forestry, geology, and landslide potential. However, image classification for physical properties of surface soils, such as strength or bearing capacity, is often obscured by other surface conditions, such as moisture and vegetation, although these are also indicators of soil strength. This project used remote methods of terrain analysis to search for areas suitable for vehicle or aircraft maneuverability based on slope, roughness, vegetation, soil type, and wetness and also performed direct classification of imagery based on soil strength. Using a maximum likelihood supervised classification approach, trained by a limited amount of ground-truth strength measurements, a soil strength classification was applied to WorldView- 2 multispectral satellite imagery. This paper presents the work done on the imagery classification for soil strength, the apparent relationship between the reflectance and soil strength, and the ongoing work to expand the technique to new imagery by using existing training sets.

DISCLAIMER: The contents of this report are not to be used for advertising, publication, or promotional purposes. Citation of trade names does not constitute an official endorsement or approval of the use of such commercial products. All product names and trademarks cited are the property of their respective owners. The findings of this report are not to be construed as an official Department of the Army position unless so designated by other authorized documents. 


\section{Contents}

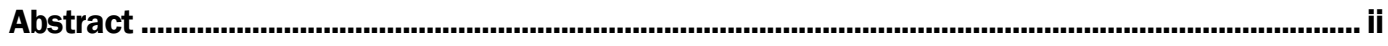

Figures and Tables.......................................................................................................................

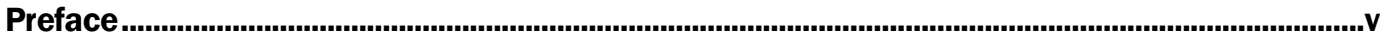

Acronyms and Abbreviation ...........................................................................................................

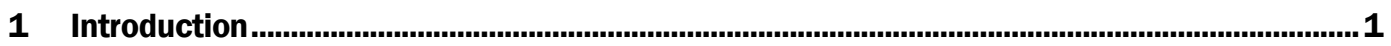

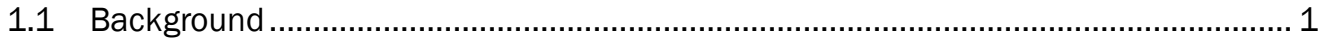

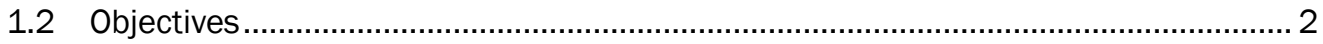

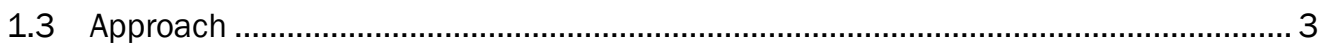

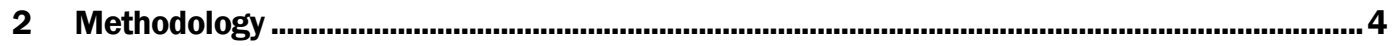

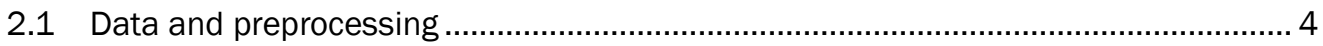

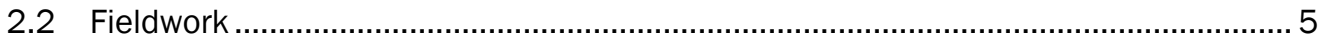

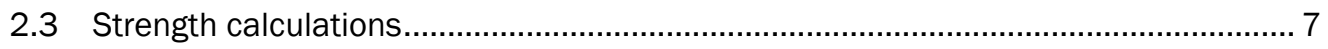

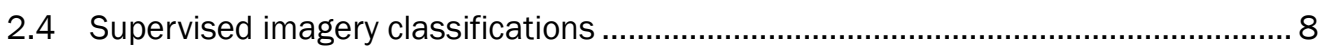

2.5 “In-scene" versus “out-of-scene" classifications ................................................. 10

2.6 Varying conditions.................................................................................... 11

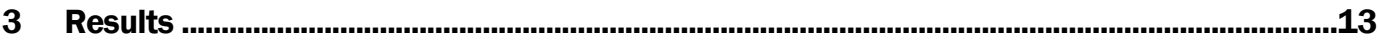

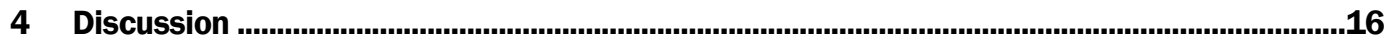

4.1 Classification case study ..................................................................... 17

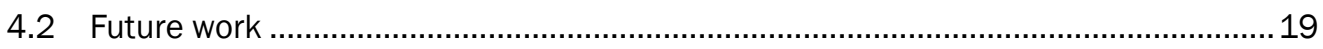

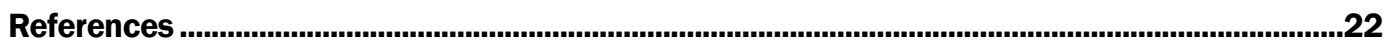

Report Documentation Page 


\section{Figures and Tables}

\section{Figures}

1 Map of the western United States (left) and close-up map of FHL field site (right) outlined in red...

2 2013, 2014, and 2015 test locations mapped on WV2 true-color composite from

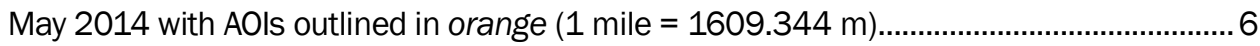

32013 WV2 image (left) and on-the-ground image (right) of the Tule landing zone............... 6

4 CRREL research technician, Jesse Stanley, taking Clegg measurements at a test location in San Miguelito ..............................................................................................

5 Example of a training ROI (yellow rectangle) surrounding a test location (orange dot) northwest of the Tule landing zone that was used in the classification of the 2013 WV2 image

6 The El Piojo reservoir and surrounding terrain in May 2013, January 2014, and March 2015, from left to right

7 WV2 true-color composites of San Miguelito test location from 2013, 2014, 2015.

8 Spectral signatures of the site outlined by the orange circle in Fig. $7 \ldots \ldots \ldots \ldots \ldots \ldots \ldots \ldots \ldots . . . .12$

9 In-scene and out-of-scene supervised classifications of the Milpitas landing zone ...........13

10 In-scene and out-of-scene supervised classifications of the El Piojo landing zone ............14

11 In-scene and out-of-scene supervised classifications of the Tule landing zone...................14

12 Average spectra of training classes 1 and 8 for the 2013, 2014, and 2015

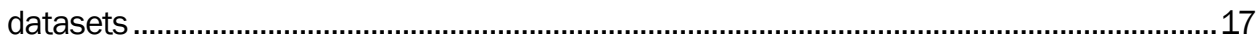

13 A compacted, high-strength area in Milpitas (M6HI) ......................................................18

14 A sandy, low-strength area in Milpitas (M6LO) ...................................................................18

15 True-color composite images of Milpitas high- and low-strength sites (top row) with the high-strength compacted area (M6HI) outlined in green and the lowstrength alluvial wash (M6LO) outlined in red. In-scene classifications of the same scene with associated legend (bottom row)

16 Field spectral measurements from high- and low-strength sites taken in 2014

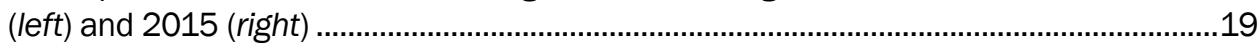

17 The difference in band wavelengths between WV2 and WV3 sensors ..............................21

\section{Tables}

1 Established convention to describe images classified using training spectra from the same scene ("in scene") versus images classified using training spectra from a different scene ("out of scene") ......................................................................................10

2 Kappa coefficients for all classified images ......................................................................15

3 Overall accuracy percentages for all classified images ...................................................15 


\section{Preface}

This study was conducted for the Army Terrestrial Environmental Modeling and Intelligence System (ARTEMIS) program under Work Item 6CJ 4D3 for the Geospatial Remote Assessment for Ingress Locations (GRAIL) project. The technical monitor was J ohn Eylander, CEERD-RR.

The work was performed by Ariana M. Sopher and Dr. Sally A. Shoop (Force Projection and Sustainment Branch, Dr. Sarah Kopczynski, Acting Chief), J esse M. Stanley, J r. (Engineering Resources Branch, J ared Oren, Chief), and Brian T. Tracy (Terrain and Ice Engineering Group, Stephen Newman, Chief), U.S. Army Engineer Research and Development Center, Cold Regions Research and Engineering Laboratory (ERDC-CRREL). At the time of publication, Dr. Loren Wehmeyer was Chief of the Research and Engineering Division, and Timothy Pangburn was Director of the Remote Sensing and Geographic Information Systems Center of Expertise (RS/GIS CX), ERDC-CRREL. The Deputy Director of ERDC-CRREL was Dr. Lance Hansen, and the Director was Dr. Robert Davis.

This research would not have been possible without the dedicated efforts of Professor Fred Kruse, Scott Runyon, Sarah Carlisle, and other supporting researchers at the Naval Postgraduate School. Stephen Newman at CRREL also offered vital guidance and support throughout this project. Andmorgan Fischer and Clint Smith at the ERDC Geospatial Research Laboratory (GRL) provided necessary expertise and fieldwork support. Furthermore, all three field campaigns would not have been possible without the support of Fort Hunter Liggett staff, including Andrew J ohnson, William Duckworth, and Range Control.

COL Bryan S. Green was the Commander of ERDC, and Dr. J effery P. Holland was the Director. 


\section{Acronyms and Abbreviation}

\begin{tabular}{|c|c|}
\hline AOI & Area of Interest \\
\hline ARTEMIS & $\begin{array}{l}\text { Army Terrestrial Environmental Modeling and Intelligence } \\
\text { System }\end{array}$ \\
\hline ASTM & American Society for Testing and Materials \\
\hline CAVIS & Clouds, Aerosols, Vapors, Ice, and Snow \\
\hline CBR & California Bearing Ratio \\
\hline CIV & Clegg Impact Value \\
\hline CRREL & Cold Regions Research and Engineering Laboratory \\
\hline DEM & Digital Elevation Model \\
\hline ENVI & Environment for Visualizing Images \\
\hline ERDC & U.S. Army Engineer Research and Development Center \\
\hline FHL & Fort Hunter Liggett \\
\hline FLAASH & Fast Line-of-Sight Atmospheric Analysis of Spectral Hypercubes \\
\hline GIS & Geographic Information System \\
\hline GRAIL & Geospatial Remote Assessment for Ingress Locations \\
\hline GRL & Geospatial Research Laboratory \\
\hline HITT & Hyperspectral Imagery Trafficability Tool \\
\hline MODTRAN & Moderate Resolution Atmospheric Transmission \\
\hline NPS & Naval Postgraduate School \\
\hline ROI & Regions of Interest \\
\hline RS/GIS CX & $\begin{array}{l}\text { Remote Sensing and Geographic Information Systems Center of } \\
\text { Expertise }\end{array}$ \\
\hline SWIR & Shortwave Infrared \\
\hline VNIR & Visible and Near Infrared \\
\hline VNIRA & Visible and Near Infrared Analysis \\
\hline WV2 & WorldView-2 \\
\hline WV3 & WorldView-3 \\
\hline
\end{tabular}




\section{Introduction}

\subsection{Background}

The ability to determine physical soil properties, such as strength or bearing capacity, is necessary for mobility and trafficability purposes; however, fieldwork is laborious, time consuming, and resource intensive. In addition, soil strength changes with time as vegetation draws moisture as well as through the seasons as the soil dries or becomes wet, freezes and expands, or thaws and saturates. Thus, the ability to map soil strength remotely will prove very useful, particularly for agencies such as the United States Army, where sending troops into the field to measure physical soil properties may be dangerous or not possible.

Recent multi- and hyperspectral satellites with high spatial resolution have allowed for tremendous growth and opportunities to study the earth from space. Remote sensing is a powerful and highly relevant tool for forecasting weather; assessing water quality; mapping terrain features; studying climate change, natural disasters, and mineralogy of the earth's surface; and many more applications. Two types of sensors are used in remote sensing. Passive sensors measure energy emissions that are naturally available, such as sunlight radiation reflected off the surface of the earth or thermal infrared radiation. Active sensors provide their own energy source by emitting radiation and collecting what is backscattered off the target.

Some previous research efforts have focused on characterizing physical soil properties by using spectral characteristics. Dutta et al. (2015) looked at mapping surface soil properties, such as chemical constituents and topsoil textural properties, by using hyperspectral data containing 224 continuous channels over a wavelength range of 400-2500 nm. Higher resolution visible and near-infrared diffuse reflectance spectroscopy has been shown to successfully characterize numerous soil properties, including texture, $\mathrm{pH}$, presence of organic and inorganic carbon, and clay mineralogy (Ge et al. 2011; Sørensen and Dalsgaard 2005). Shepherd and Walsh (2002) estimated soil properties from soil reflectance spectra by using a spectral library of over 1000 topsoils from eastern and southern Africa. Ben-Dor et al. (2002) analyzed spectral laboratory data that showed significant capability to predict soil field moisture, soil saturated moisture, and soil salinity by using visible and near infrared analysis (VNIRA). Ben- 
Dor et al. (2002) developed a procedure to successfully map soil properties of Izrael Valley in northern Israel by using hyperspectral imagery from an airborne sensor. In 2003, Ben-Dor et al. conducted a study on determining properties of soil structural crusts solely from spectral information in the $1.2-2.5 \mu \mathrm{m}$ wavelength range.

There have also been numerous efforts to develop algorithms and processes to successfully map soil properties from imagery. Pass et al. (n.d.) developed Revelatum ${ }^{\mathrm{TM}}$, a set of algorithms and visualizations focused on revealing patterns and relationships in multispectral (and other) imagery to improve decision-making and to offer predictive capabilities. Bachmann et al. (2006) explored the use of manifold coordinate estimation algorithms, which were typically used outside of the remote-sensing field, on hyperspectral imagery to discriminate between land-cover types with a high level of spectral similarity. In 2012 at the Naval Research Laboratory, Bachmann et al. developed the Hyperspectral Imagery Trafficability Tool (HITT), a geospatial analysis tool that creates estimated soil bearing strength maps from hyperspectral data taken in coastal regions. Between 2006 and 2007, Boeing developed a method to predict soil type from multispectral imagery and digital elevation data (Hines and Wolboldt 2008). This area of study has received increased attention because remote sensing of soil spectral characteristics to determine soil physical properties is nondestructive to the soil, rapid, and less expensive than conventional soil analytical techniques (Viscarra Rossel et al. 2006).

\subsection{Objectives}

The work described in this report was funded under the Army Terrestrial Environmental Modeling and Intelligence System-Geospatial Remote Assessment for Ingress Locations (ARTEMIS-GRAIL) research program. The long-term goal of this program is to automate a process to locate austere landing zones and drop zones by using satellite imagery and remote-sensing data. Before landing an aircraft on an unpaved and austere surface, personnel must confirm that the site passes numerous requirements. Among other things, the site must be flat, large enough, and free from any obstructions and must possess soil properties suitable to withstand the proposed applied loads. Because of its strong background in soil strength testing, mobility mapping, and remote sensing, the U.S. Army Engineer Research and Development Center Cold Regions Research and Engineering Laboratory (ERDC-CRREL) is able to further research soil strength prediction based on satellite imagery. 
Soil strength is a complicated soil property affected by numerous factors, including soil density, soil type, moisture content, plasticity characteristics, gradation, and particle shape. This report explores remote soil strength prediction in a geographic information system (GIS) environment, one objective of the ARTEMIS-GRAIL program.

\subsection{Approach}

This study used supervised classification techniques on multispectral satellite imagery to classify areas of higher soil strength versus lower soil strength. Standard image classification algorithms were run using a GIS platform; and all images were classified into ten classes based on soil strength, water, and vegetation. Specific pixels corresponding to areas of known soil strength were selected to train the algorithms to predict strength across the entire image.

This study used multispectral satellite imagery from a passive sensor for all remote-sensing analysis. Multispectral platforms collect imagery at multiple (on the order of ten) wavelength bands across the electromagnetic spectrum. Multispectral imagery is generally used in analyses of terrain and atmospheric features such as vegetation, snow, and clouds; detection of changes in terrain or atmospheric conditions over time; classification of data from the images into categories of objects on the ground; and other applications.

Classification is the process of categorizing each pixel into a certain spectral class to describe what kind of object that pixel depicts. Supervised classifications use training data, which are groups of pixels that the analyst knows to be of a certain information class, or characteristic on the ground. A common and important assumption that is typically made in remote sensing for statistical supervised classifications is that each spectral class can be described by some probability distribution in multispectral space (Richards 1999). In the current study, pixels used to train the classification algorithms were selected based on soil strength data collected in the field. Numerous soil strength measurements were taken throughout the study area while multispectral imagery was collected. Pixels designating areas of known strength were then used to train a classification of the entire image. Fieldwork with subsequent imagery analysis was executed in 2013, 2014, and 2015; and results over the three years were compared. 


\section{Methodology}

A broad overview of the explored methodology is as follows:

1. Sample soil strength at numerous and varied locations during concurrent satellite imagery collections.

2. From the imagery, create training regions of interest (ROIs) at each measurement location and group into categories of strength.

3. Use spectra of pixels within the ROIs to train a supervised "strengthbased" classification of the entire image.

4. Explore whether training classes from one image can be applied to other images.

5. Evaluate the success of the mapping.

\subsection{Data and preprocessing}

This study used Digital Globe's WorldView-2 (WV2) 8-band multispectral satellite imagery. This earth-imaging satellite was launched in 2009 as the first high-resolution, commercially available satellite and offers $1.85 \mathrm{~m}$ multispectral resolution (Digital Globe 2016). WV2 multispectral bands range from 0.4 to $1.04 \mu \mathrm{m}$ and are typically used for vegetation, coastal, and land-use applications and mapping (Kruse 2015). Over the course of this study, three WV2 collections of approximately $880 \mathrm{~km}^{2}$ over Fort Hunter Liggett (FHL) in southern Monterey County, CA, were acquired from Digital Globe. The collections took place in May 2013, J anuary 2014, and March 2015. Figure 1 shows the location of FHL within California.

Figure 1. Map of the western United States (left) and close-up map of FHL field site (right) outlined in red.
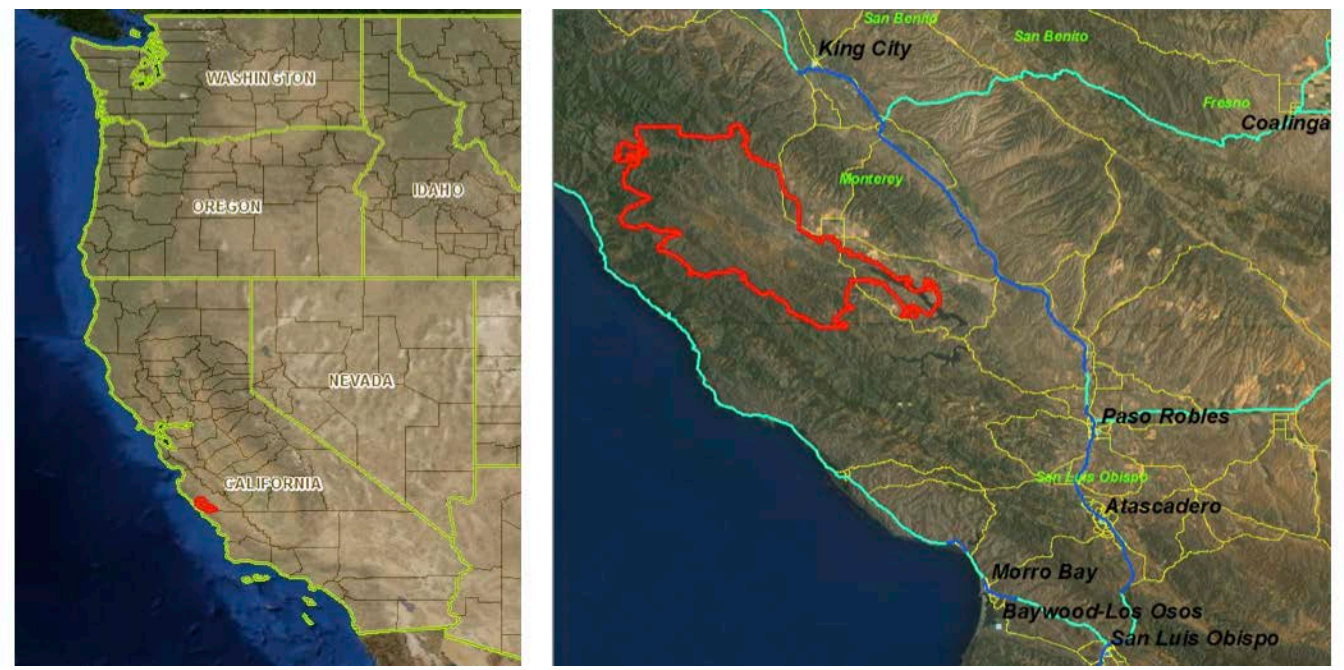
The Naval Postgraduate School (NPS), under research professor Fred A. Kruse, preprocessed the imagery. The three datasets were orthorectified and corrected to reflectance. Orthorectification required use of a coincident digital elevation model (DEM) and a high-resolution orthophotograph collected by the National Agriculture Imagery Program. Ground control points were selected to constrain the orthorectification (Kruse 2015). The three images were scaled to radiance and then corrected to reflectance by using the moderate resolution atmospheric transmission (MODTRAN) atmospheric compensation model and software developed by Spectral Sciences, Inc., called Fast Line-of-Sight Atmospheric Analysis of Spectral Hypercubes (FLAASH) (Kruse 2015). The fraction of incident light reflected by some surface is described by reflectance, which is a unitless number between zero and one (Shaw and Burke 2003).

On the occasions when field spectral measurements were also taken during site visits, the imagery was further modified to match measured reflectance of specific target sites by using an empirical line correction method (Kruse 2015). The above-described efforts of NPS resulted in three coregistered and calibrated WV2 reflectance images from J une 2013, J anuary 2014, and March 2015.

\subsection{Fieldwork}

During each visit to FHL, team members from CRREL and other collaborating organizations, such as NPS and the ERDC Geospatial Research Laboratory (GRL), measured soil strength at various locations within the image collection area by using a Clegg Impact Hammer, a dynamic cone penetrometer, and a static cone penetrometer. Moisture content and soil density were also measured using a FieldScout TDR 300 Moisture Meter and a Troxler soil density gauge, respectively. Exact sampling locations were marked using a handheld global positioning system. Furthermore, in 2014 and 2015, field spectral measurements were taken using an ASD FieldSpec4 Standard-Res spectroradiometer. Reflectance measurements from 0.4 to $2.5 \mu \mathrm{m}$ with 3-10 $\mathrm{nm}$ of spectral resolution were collected at target locations around FHL with the help of NPS and GRL personnel. Spectra of specific materials like rocks, vegetation, and soil were taken as were walking average measurements to illustrate each test location on a remotesensing scale. 
Fieldwork focused on five general geographic areas of interest (AOIs): Milpitas, J olon, San Miguelito, Tule, and El Piojo. These areas covered a variety of terrain types and were chosen to include a pre-existing landing zone or drop zone or terrain suitable for one. Figure 2 shows the locations of the five AOIs throughout FHL, denoted with orange rectangles, as well as the numerous test locations, denoted with small dots. Figure 3 shows an example from the satellite imagery of one of the existing landing zones.

Figure 2. 2013, 2014, and 2015 test locations mapped on WV2 true-color composite from May 2014 with AOls outlined in orange $(1$ mile $=1609.344 \mathrm{~m})$.

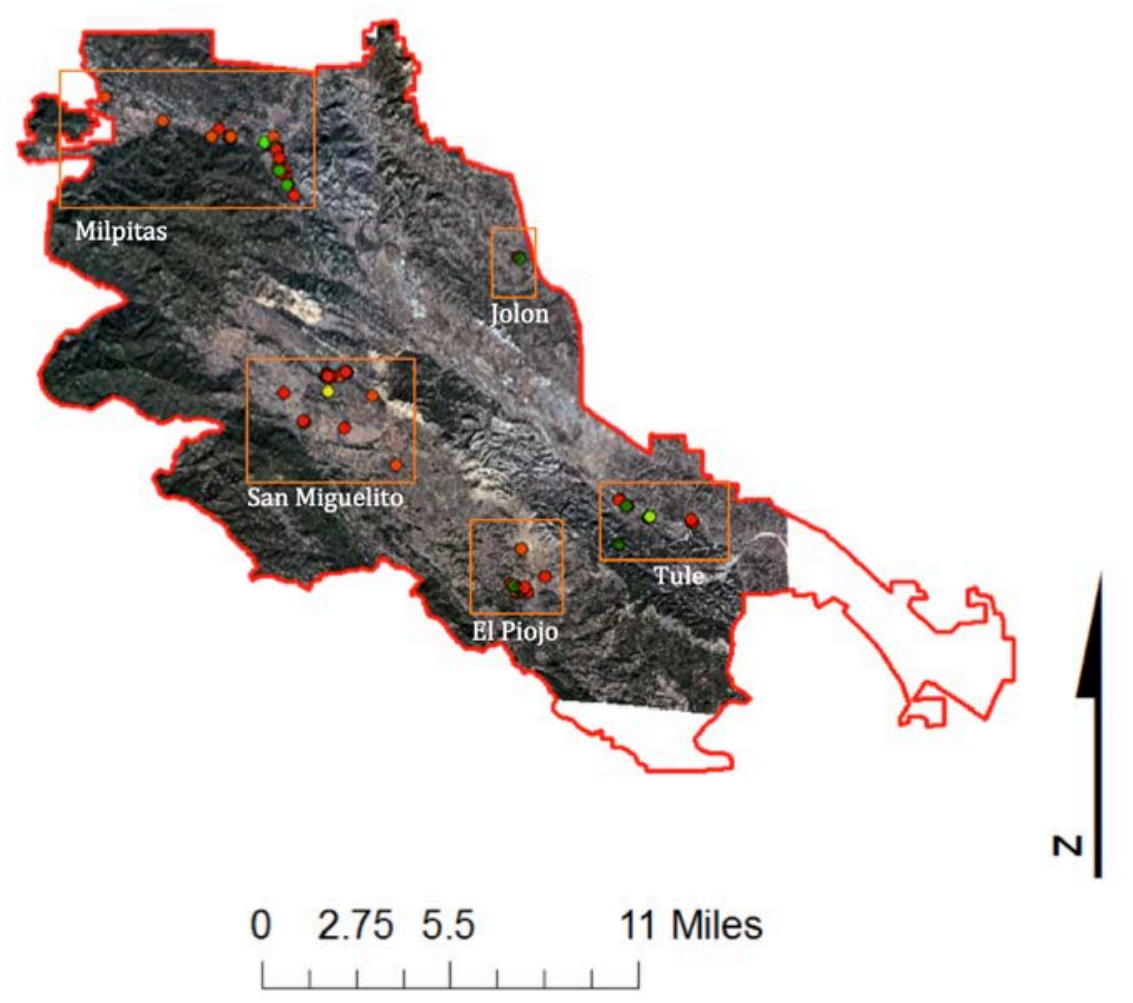

Figure 3. 2013 WV2 image (/eft) and on-the-ground image (right) of the Tule landing zone.
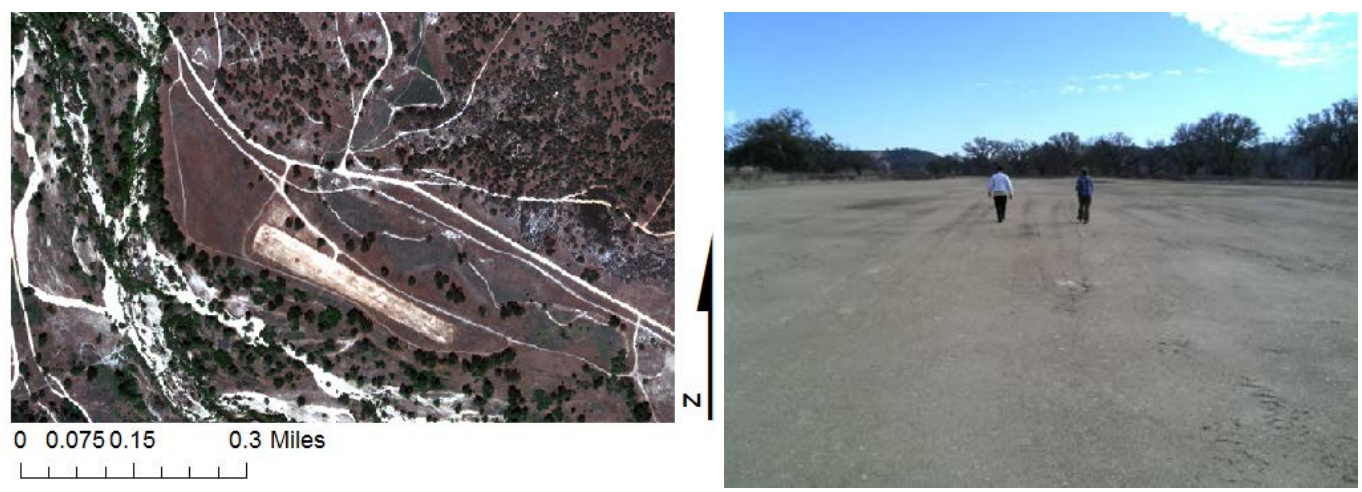


\subsection{Strength calculations}

The California bearing ratio (CBR) is a measurement of soils' load-bearing capacity and is commonly used to test subgrades and base layers of airfield and road pavements. The CBR test was created by the California State Highway Department and was integrated into the design of flexible pavements by the Army Corps of Engineers in the 1940s (Middlebrooks and Bertram 1950). The American Society for Testing and Materials (ASTM) and international standards describe proper CBR measurement technique (ASTM 2005; Al-Amoudi et al. 2002). CBR is described by the ratio of the pressure required to penetrate the soil of interest compared to the pressure for equal penetration of crushed well-graded limestone. This ratio is multiplied by 100, and CBR is reported as a percentage. Despite its widespread use, laboratory and field CBR testing is laborious and time intensive. As such, researchers have looked for more simple and portable techniques that correlate well with the CBR test. One of these methods uses the Clegg Impact Hammer, which is light, easy to operate, and cost effective. The Clegg Impact Hammer measures stiffness of the soil surface by dropping a standard weight equipped with a piezoelectric accelerometer from a constant height and is shown in Figure 4. The peak rate of rebound of the hammer is recorded (Al-Amoudi et al. 2002).

Figure 4. CRREL research technician, Jesse Stanley, taking Clegg measurements at a test location in San Miguelito.

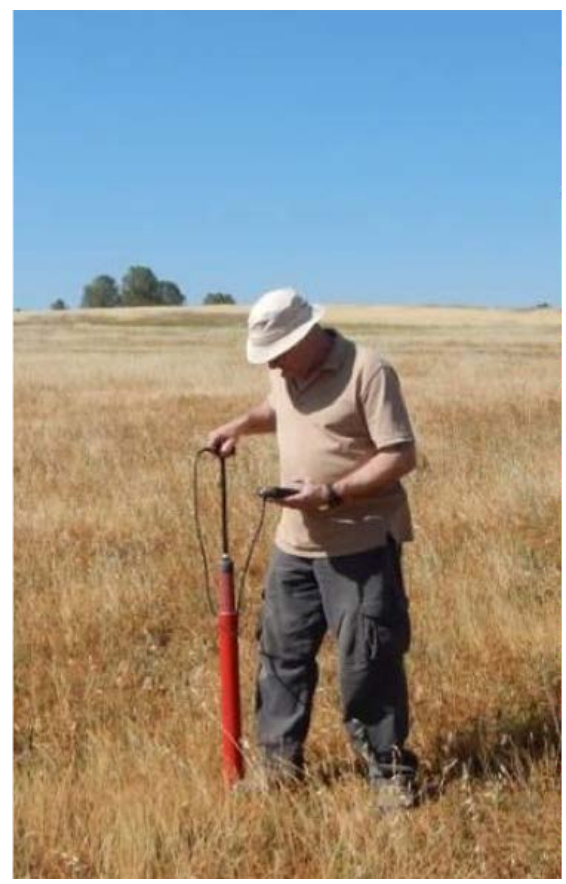


In this experiment, CBR was calculated from measurements taken in the field with a $2.25 \mathrm{~kg}$ Clegg Impact Hammer by using the following equation published in 2011 by Dr. Baden Clegg Pty Ltd. for the Dublin Light Rail Transit Project (Clegg 2011):

$$
C B R=e^{(10 * C I V-14.936) / 79.523}
$$

where CIV (Clegg impact value) is the third out of four drops of the Clegg Impact Hammer.

At each test location, three Clegg measurements were taken; and an average calculated CBR was used in subsequent analysis as a measure of that site's soil strength.

Based on the average CBR value, each site was assigned a "strength class" by using the following conditions:

- Class 1: $0 \%<\mathrm{CBR} \leq 5 \%$

- Class 2: $5 \%<\mathrm{CBR} \leq 10 \%$

- Class 3: $10 \%<\mathrm{CBR} \leq 15 \%$

- Class 4: $15 \%<\mathrm{CBR} \leq 20 \%$

- Class 5: $20 \%<\mathrm{CBR} \leq 25 \%$

- Class 6: $25 \%<\mathrm{CBR} \leq 30 \%$

- Class 7: $30 \%<\mathrm{CBR} \leq 35 \%$

- Class 8: 35\% <CBR

\subsection{Supervised imagery classifications}

Coordinates for each test location were selected in large $(10 \times 10 \mathrm{~m})$ open areas of homogenous terrain. After collecting strength data at these locations in the field, ROIs were manually drawn on the WV2 imagery encapsulating about nine pixels of homogenous terrain surrounding each test location, as shown in Figure 5. The reflectance values of these pixels are called "training data." During the classification, training data is compared with reflectance values of every other pixel in the image to determine its most appropriate class. While there is uncertainty associated with using one strength measurement for each $3 \times 3$ pixel $(5.5 \times 5.5 \mathrm{~m}$ on the ground) ROI, it was assumed that the conditions and properties of the soil surrounding each test location varied at greater scales than the area of the ROI polygons. 
The reflectance values of each training pixel were used to train a supervised classification of the entire image by using the maximum likelihood algorithm. ROIs encapsulating pixels indicating heavy vegetation and water were also created and incorporated into the supervised classification.

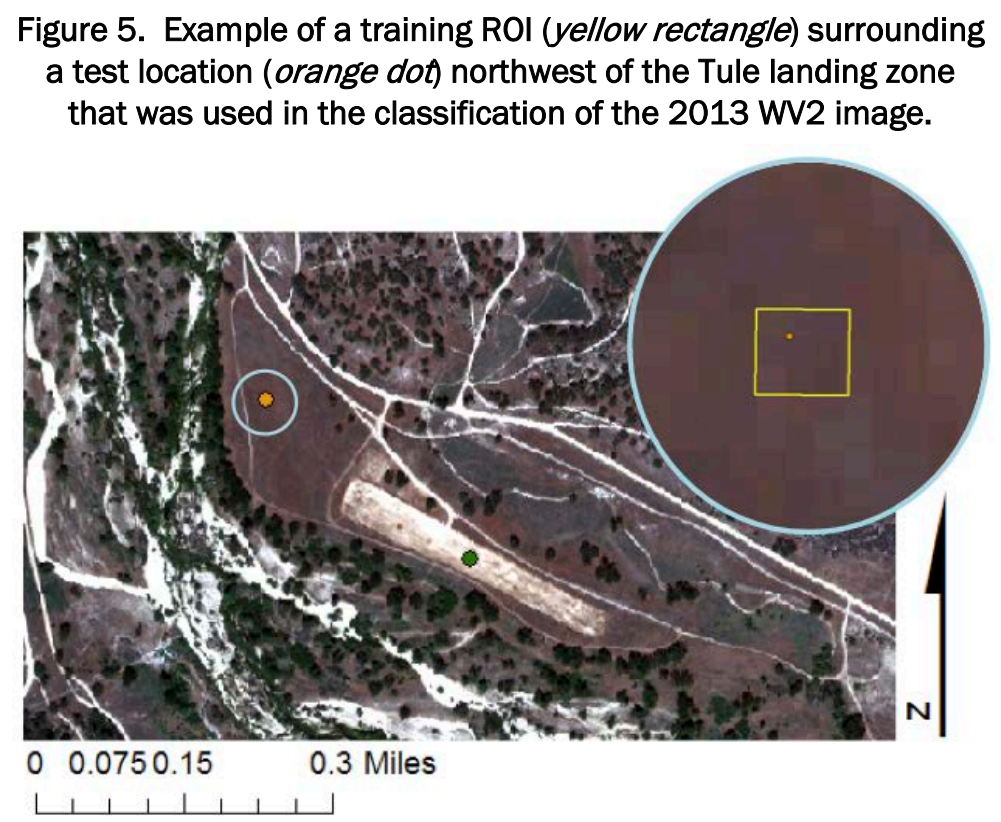

The maximum likelihood algorithm is the most common supervised classification technique used with remote-sensing image data (Richards 1999). This algorithm estimates a normal probability distribution from sufficient training data to describe the chance, or probability, that some pixel belongs to a designated class. For each pixel at a given position in multispectral space, a set of probabilities is determined to define the relative likelihood that that pixel belongs to each class (Richards 1999). In this example, there are 8 soil classes based on strength plus a vegetation and a water class, resulting in 10 classes total. The soil classes were determined by CBR, as discussed previously.

Supervised classifications were run in the Environment for Visualizing Images (ENVI) software application, which uses the following discriminant function to calculate the probability of a pixel belonging to a specific class when applying the maximum likelihood algorithm (Richards 1999; Harris Geospatial Solutions 2016b):

$$
g_{i}(x)=\ln p\left(\omega_{i}\right)-1 / 2 \ln \left|\Sigma_{i}\right|-1 / 2\left(x-m_{i}\right)^{T} \Sigma_{i}^{-1}\left(x-m_{i}\right)
$$


where

$$
\begin{aligned}
\mathrm{i}= & \text { the class, } \\
\mathrm{x}= & \mathrm{n} \text {-dimensional data (where } \mathrm{n} \text { is the number of bands) } \\
\mathrm{p}\left(\omega_{\mathrm{i}}\right)= & \text { the probability that class } \omega_{\mathrm{i}} \text { occurs in the image and is } \\
& \text { assumed the same for all classes, } \\
\left|\Sigma_{i}\right|= & \text { the determinant of covariance matrix of the data in class } \omega \mathrm{i}, \\
\Sigma^{-1}= & \text { its inverse matrix, and } \\
m_{i}= & \text { the mean vector. }
\end{aligned}
$$

\section{5 "In-scene" versus "out-of-scene" classifications}

After running the aforementioned methodology on WV2 imagery from 2013,2014 , and 2015, the authors explored whether spectra from one image could be used as training spectra to classify a different image. Atmospheric, terrain, and vegetation conditions vary between collections. Furthermore, the presence of the atmosphere obscures incident radiation on the surface of the earth and also attenuates reflected radiation. However, all three images were atmospherically corrected, and all analysis remained site specific. The ability to classify an image collected in 2015 with spectra from an image of the same scene collected at a different time, and ulti-

\begin{tabular}{|c|c|c|c|}
\hline & 2013 WV2 Image & 2014 WV2 Image & 2015 WV2 Image \\
\hline 2013 Training Spectra & "In Scene" & "Out of Scene" & "Out of Scene" \\
\hline 2014 Training Spectra & "Out of Scene" & "In Scene" & "Out of Scene" \\
\hline 2015 Training Spectra & "Out of Scene" & "Out of Scene" & "In Scene" \\
\hline
\end{tabular}
mately a different location, would further minimize the need for "boots on the ground." Table 1 illustrates the combinations used to train and classify the image.

Table 1. Established convention to describe images classified using training spectra from the same scene ("in scene") versus images classified using training spectra from a different scene ("out of scene").

Training ROIs created previously for in-scene classifications were used to select training spectra for out-of-scene classifications. The maximum likelihood algorithm was used to run all nine of the classifications depicted in the matrix in Table 1. 


\subsection{Varying conditions}

Because this analysis was conducted over the course of three years and visits to the test site were timed to capture different seasonal conditions, terrain and climate conditions were noticeably different during each visit and were apparent in the imagery. This phenomenon can be seen in Figure 6, which shows one location for each of the image collection dates.

Figure 6. The El Piojo reservoir and surrounding terrain in May 2013, January 2014, and March 2015, from left to right.
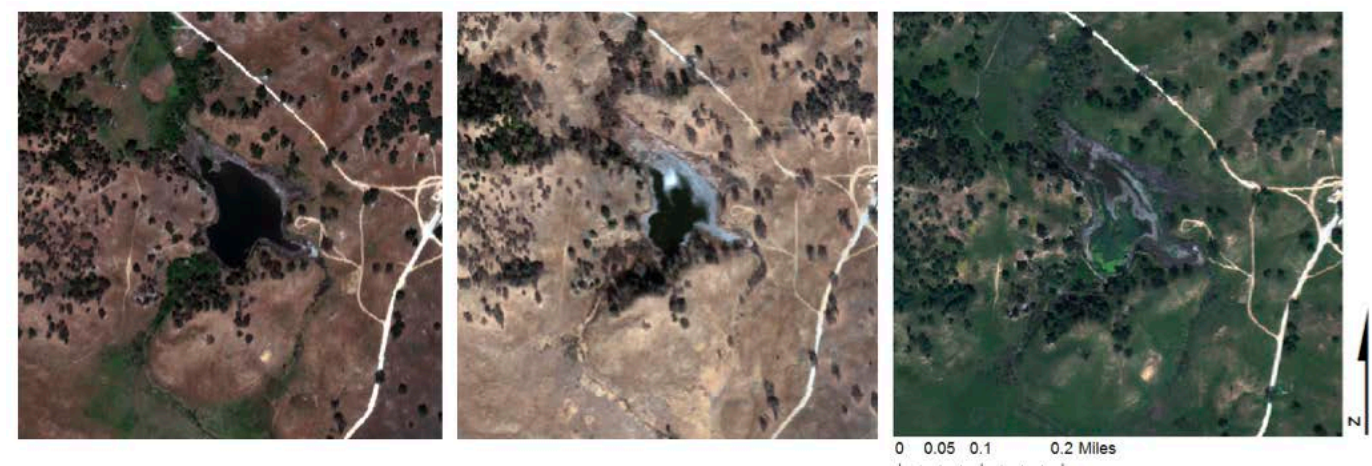

J anuary 2014 was the driest of the three visits whereas March 2015 was the most vegetated and moist. As such, areas of bare soil or dried grass in 2014 were mostly covered in healthy vegetation in 2015.

Figure 7 shows the WV2 imagery over San Miguelito from each year. Spectral signatures of the test location outlined in an orange circle in Figure 7 are shown in Figure 8. The signatures are more indicative of healthy vegetation in 2013 and particularly in 2015 while the 2014 signature is more indicative of bare soil.

Figure 7. WV2 true-color composites of San Miguelito test location from 2013, 2014, 2015.
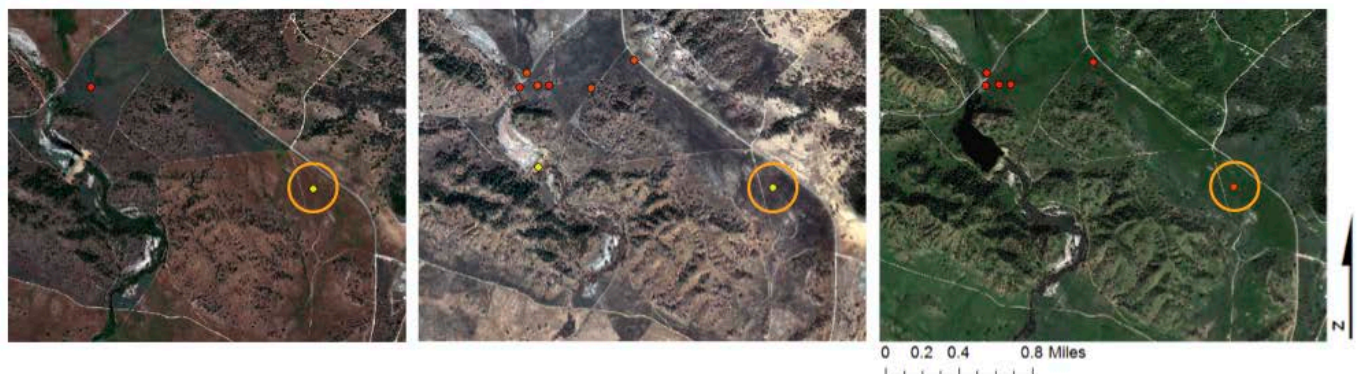
Figure 8. Spectral signatures of the site outlined by the orange circle in Fig. 7.

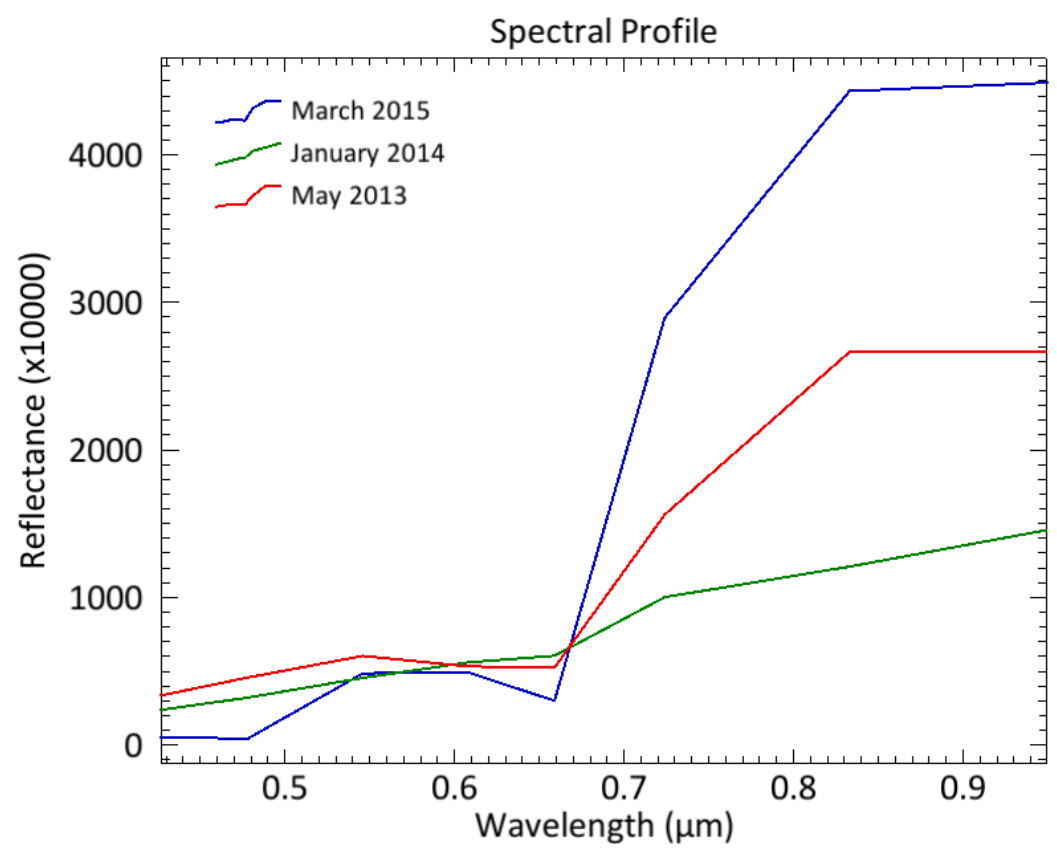




\section{Results}

Figures 9, 10, and 11 show examples of all nine training and classifications combinations at three different landing zones (Milpitas, El Piojo, and Tule, respectively).

To analyze the success of each classified image, kappa coefficients $(\kappa)$ and overall accuracy percentages were calculated using an ENVI post-processing tool called Confusion Matrix Using Ground Truth ROIs. This tool compares the assigned class of pixels in each ground-truth ROI with the class of those pixels in the resulting classified image.

The kappa coefficient is a measurement used to quantify agreement between two or more entities and takes into account that agreement may occur purely by chance. The kappa coefficient returns a value from 0 to 1 where 0 signifies no agreement and 1 signifies perfect agreement and is calculated as follows (Sim and Wright 2005):

$$
\kappa=\frac{\text { observed agreement-chance agreement }}{1-\text { chance agreement }}
$$

Kappa coefficients and overall accuracy calculations for all nine classification combinations are given in Tables 2 and 3, respectively.

Figure 9. In-scene and out-of-scene supervised classifications of the Milpitas landing zone.

Milpitas LZ
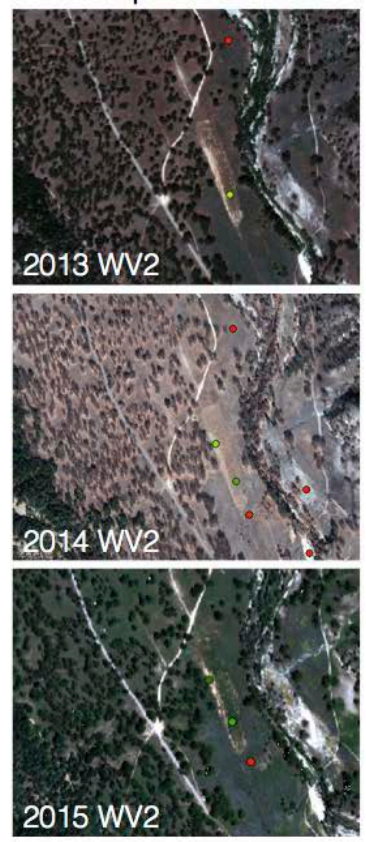

2013 image

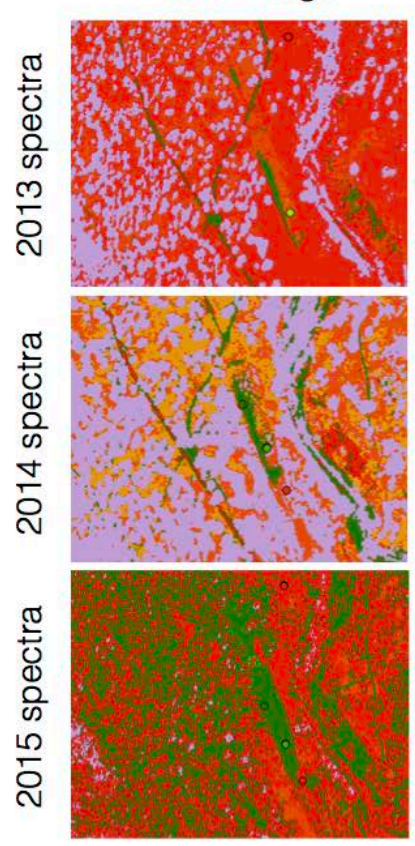

2014 image

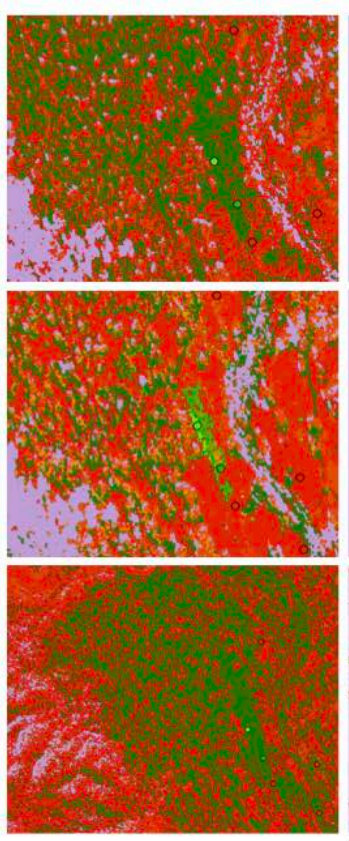

2015 image

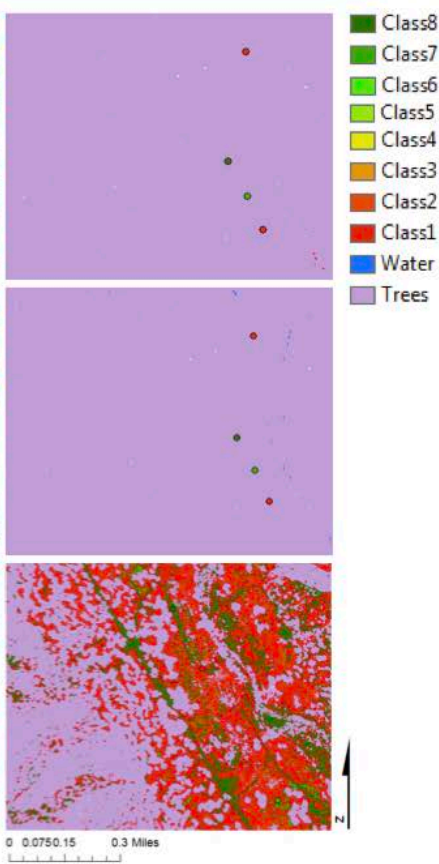


Figure 10. In-scene and out-of-scene supervised classifications of the El Piojo landing zone.

El Piojo LZ
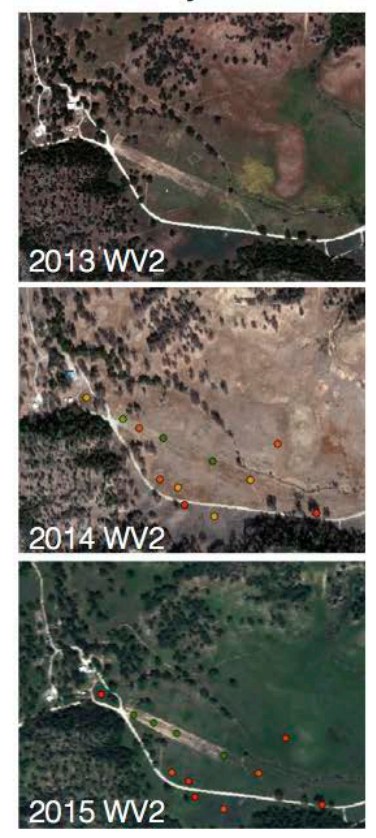
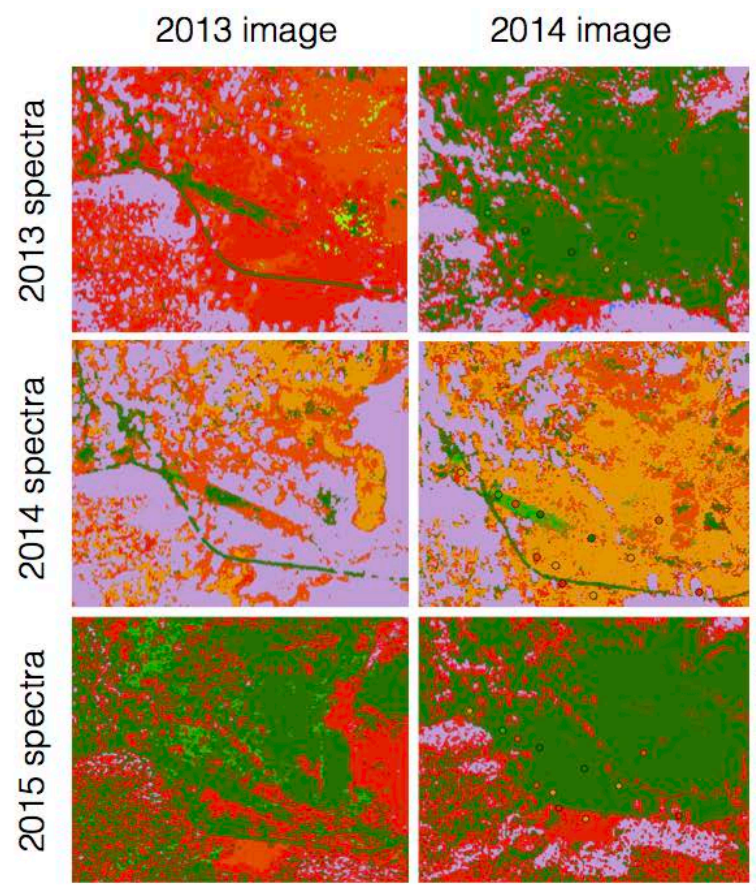

2015 image

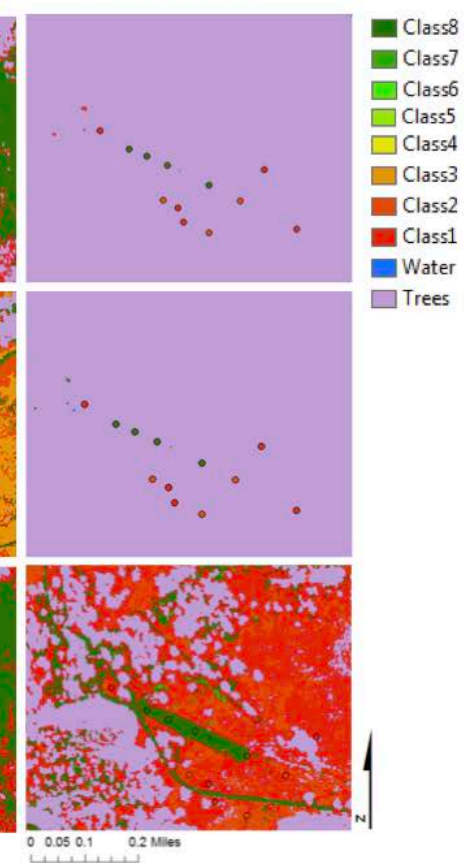

Figure 11. In-scene and out-of-scene supervised classifications of the Tule landing zone.

Tule LZ
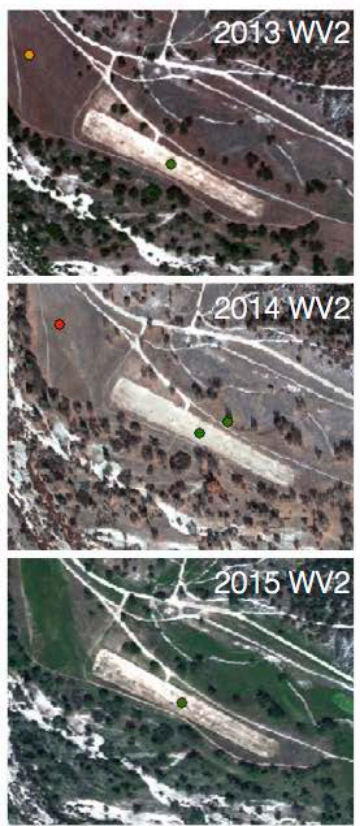

2013 image
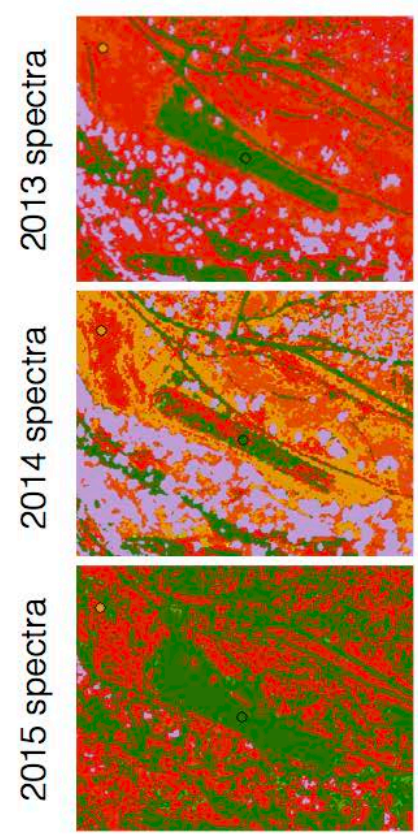

2014 image
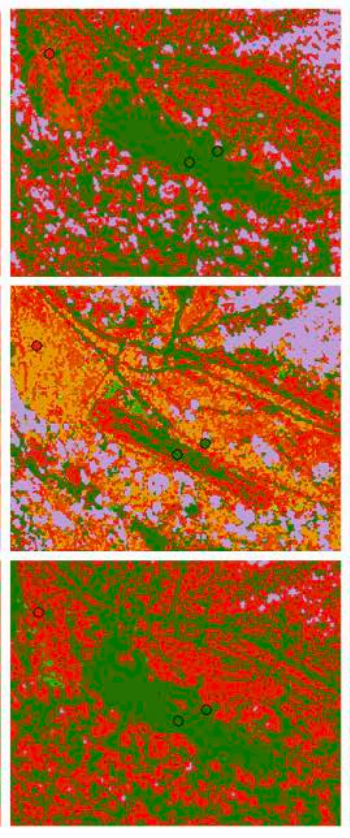

2015 image

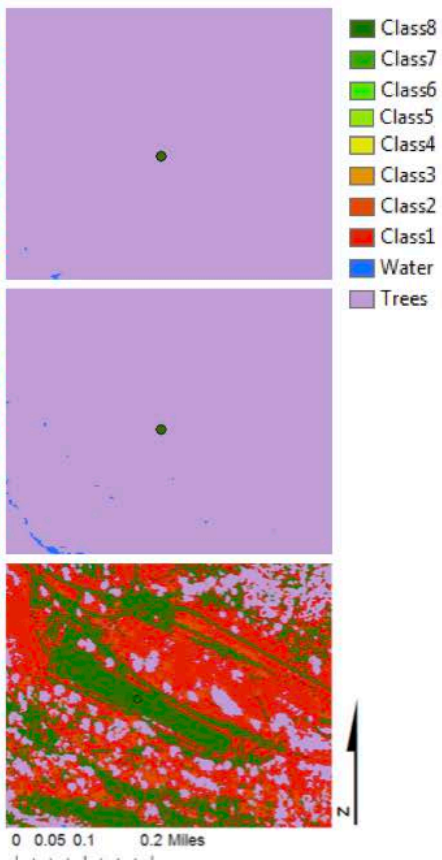


Table 2. Kappa coefficients for all classified images.

\begin{tabular}{l|c|c|c|}
\multicolumn{1}{c}{} & \multicolumn{2}{c}{ 2013 WV2 Image } & \multicolumn{2}{c|}{ 2014 WV2 Image } & WV2 Image \\
\cline { 2 - 4 } 2013 Training Spectra & 0.8835 & 0.0672 & 0.0000 \\
\cline { 2 - 4 } 2014 Training Spectra & 0.0974 & 0.7345 & 0.0000 \\
\cline { 2 - 4 } 2015 Training Spectra & 0.2495 & 0.0256 & 0.8655 \\
\cline { 2 - 4 } & &
\end{tabular}

Table 3. Overall accuracy percentages for all classified images.

\begin{tabular}{l|c|c|c|}
\multicolumn{1}{c}{} & \multicolumn{2}{c}{2013 WV2 Image } & \multicolumn{2}{c}{2014 WV2 Image } & \multicolumn{1}{c}{2015 WV2 Image } \\
\cline { 2 - 4 } 2013 Training Spectra & $91.72 \%$ & $36.40 \%$ & $0.00 \%$ \\
\cline { 2 - 4 } 2014 Training Spectra & $37.66 \%$ & $78.83 \%$ & $0.00 \%$ \\
\cline { 2 - 4 } 2015 Training Spectra & $54.58 \%$ & $36.34 \%$ & $90.43 \%$ \\
\cline { 2 - 4 } & &
\end{tabular}

Pixels used to train the classification algorithms were also used as groundtruth data to determine the above results. It would be more accurate to use a percentage of fieldwork CBR measurements to create training data and run the classifications and to use the rest of the data to verify classification results. Section 4.2 further discusses details on this topic. 


\section{Discussion}

In-scene classifications did, in general, correctly classify most existing roads and compacted landing zones as high strength. Naturally, out-ofscene classifications had much lower kappa values than in-scene classifications, particularly when 2013 and 2014 spectra were used to train a classification of the 2015 image.

Out-of-scene classifications of the 2015 images resulted in nearly all of the pixels classifying as vegetation because, during that collection, the vegetation was very healthy and any significant amount of vegetation dominated the spectral profile. Thus, pixels corresponding to bare soils during drier years should not be used to train analysis of sites with widespread vegetation in more moist years.

It is also important to note that the out-of-scene classification methodology relies not only on similar terrain and climate conditions but also on similar atmospheric conditions, which are by no means constant. Thus, the methodology is heavily reliant on preprocessing to correct for those varying conditions. However, preprocessing correction algorithms of multispectral data to reflectance are not able to perfectly calibrate for these varying conditions, even when imagery is processed using scene-specific parameters.

Although differences between spectral signatures and physical soil characteristics like strength are subtle, in-scene strength-based classifications may show some utility. As previously mentioned, any presence of vegetation will dominate the spectral signature, which makes it more difficult to understand spectral characteristics related to physical soil properties. High-strength training data (Class 8) were mostly taken on landing zones and roads. The average signature of Class 8 training data is noticeably different from the average signature of lower-strength training data, as seen in Figure 12. This is likely because the areas of very high-strength soil surfaces are engineered and modified from their natural state. Dirt roads and most landing zones were scraped, leveled, and compacted. The scraped surfaces are likely the cause of the noticeable difference in the averaged spectral profiles, as these scraped surfaces reflect more intensely. Differences in spectra amongst strength classes 1- 7 are subtler. 
Figure 12. Average spectra of training classes 1 and 8 for the 2013, 2014, and 2015
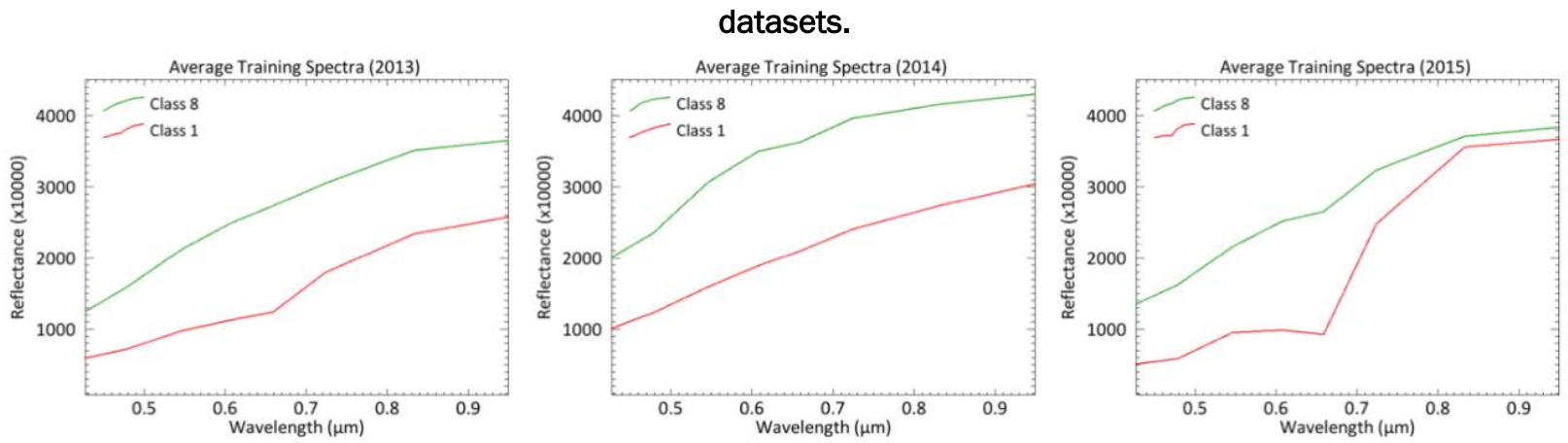

Overall, in-scene classifications have a much higher chance of success because the training data and the images are temporally consistent. This is verified in Tables 2 and 3. Variation among years due to increased precipitation, human modification of the land surface, or changing atmospheric conditions will not impact the success of in-scene classifications but may have a drastic effect on out-of-scene classifications. More statistical analysis should, however, be done to compare the measured field spectra, the WV2 training spectra, and the physical properties of the soil. Further analysis would illuminate any statistically significant correlation between the reflectance properties and physical properties at this spatial and spectral remote-sensing resolution. In addition, the benefits of classifying the most-critical components (highest strength) could outweigh erroneous classifications of terrain not otherwise suitable (i.e., too vegetated, too weak, or too wet).

\subsection{Classification case study}

Comparing two test locations in the Milpitas AOI that have very different physical properties but have similar spectral reflectance illustrates the strengths and limitations of this strength-based classification technique. One of the sites is a compacted and leveled dirt area while the other is a sandy alluvial wash. These sites are referred to as M6HI and M6LO and are depicted in Figure 13 and 14 respectively.

Unsupervised classification techniques were originally tested on this dataset; however, pixels with similarly high reflectance values but differing physical properties were often misclassified. Figure 15 shows in-scene supervised classifications of this area. M6HI was typically classified as high strength in the WV2 classifications. M6LO was classified as low strength in 2013; however, in 2015, M6LO was mostly classified incorrectly as high strength. Regions of low-strength alluvial wash, such as M6LO, or high- 
strength scraped and compacted dirt runways or parking areas, such as M6HI, can be useful indicators of the success of such a technique.

Figure 13. A compacted, high-strength area in Milpitas (M6HI).
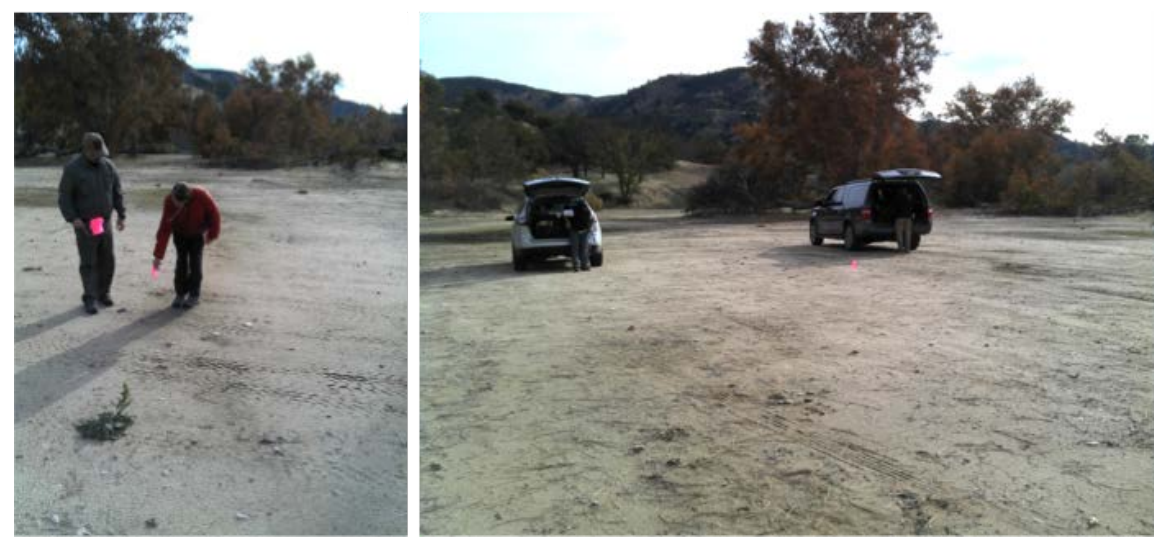

Figure 14. A sandy, low-strength area in Milpitas (M6LO).
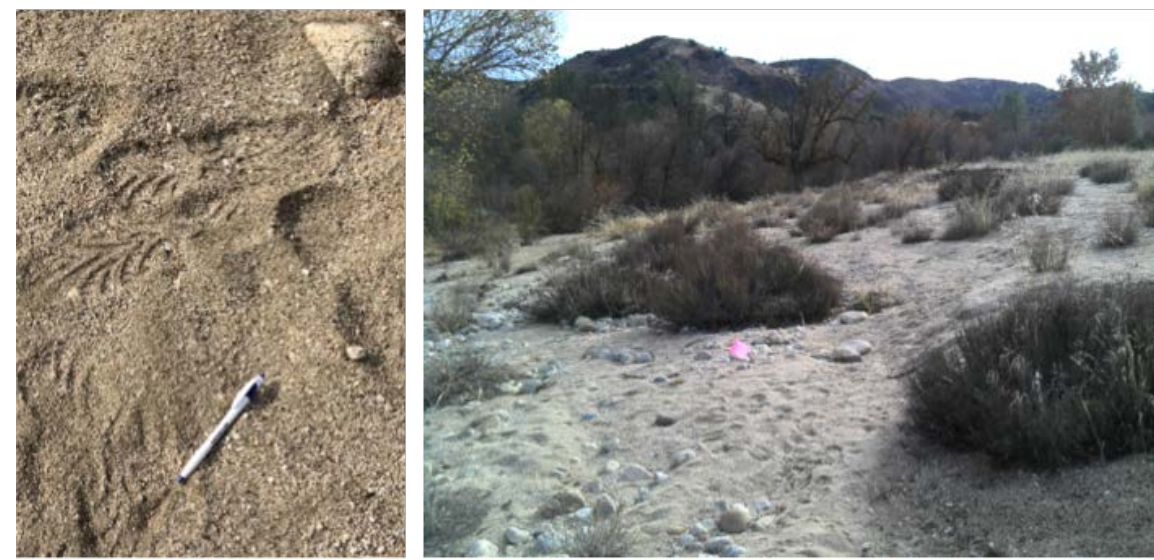

Figure 15. True-color composite images of Milpitas high- and low-strength sites (top row) with the high-strength compacted area (M6HI) outlined in green and the low-strength alluvial wash (M6LO) outlined in red. In-scene classifications of the same scene with associated legend (bottom row).
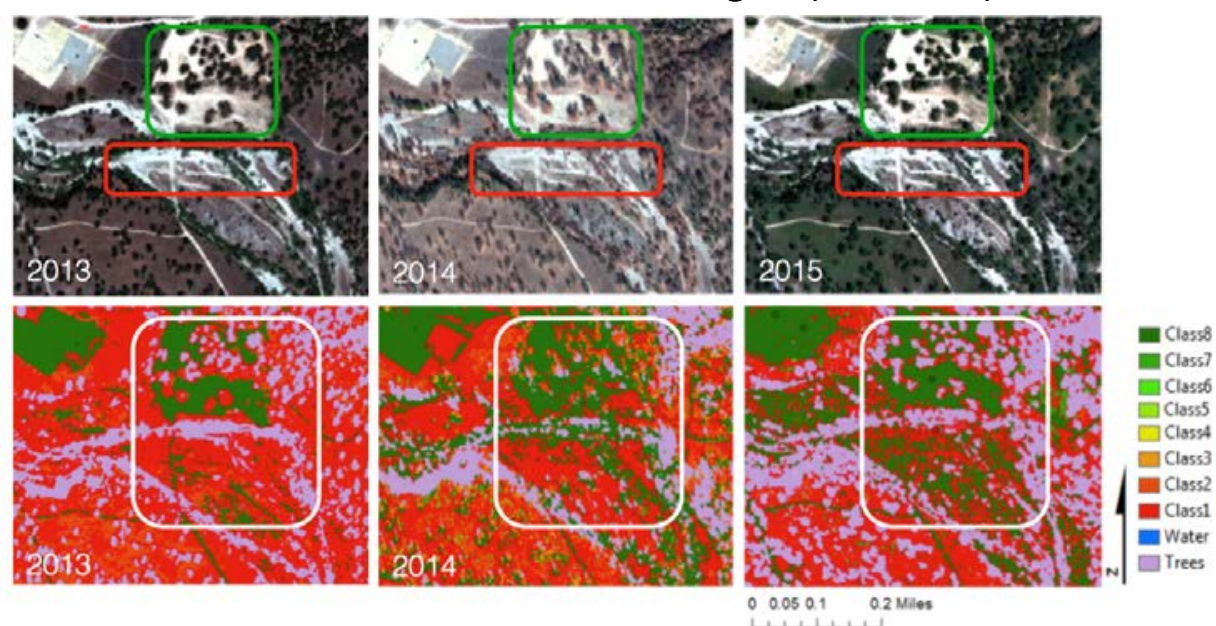
Field spectral measurements taken at these two sites in 2014 and 2015 show similar shapes and levels of reflectance. However, as seen in Figure 16 , both spectra were much brighter, and the relative brightness was reversed in 2015.

Figure 16. Field spectral measurements from high- and low-strength sites taken in 2014 (/eft) and 2015 (right).
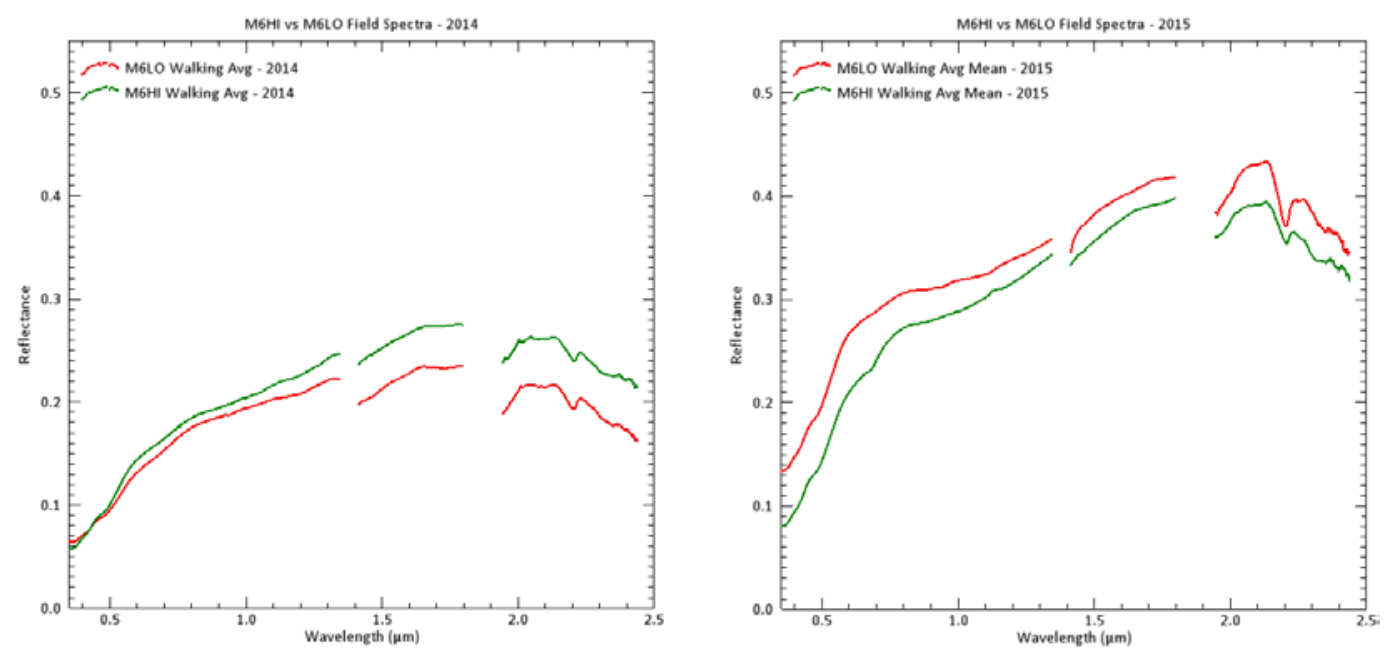

It is possible that increased moisture in the soil at $\mathrm{M} 6 \mathrm{HI}$ is the reason for this reversal of relative brightness in 2015. The volumetric water content of M6HI was three times that of M6LO in 2015, likely because fine-grained compacted soil holds moisture much more than the well-drained, sandy soil seen at M6LO. Increased and healthier vegetation in 2015 may be the reason that many more pixels in this alluvial wash region surrounding M6LO were misclassified as high strength.

\subsection{Future work}

To extend this work, it may be helpful and interesting to apply the same methodology on a more dry and barren test site to analyze spectral properties of bare soils without any presence of vegetation. Focusing just on bare soil would allow for a more detailed study of the relationship between soil physical properties and reflectance.

Extension of this work should also include a thorough analysis of the field spectra taken in J anuary 2014 and March 2015 and a more recent set of field spectra collected in December 2015 and planned for J uly 2016. A statistical comparison of field spectra, imagery spectra, and measured physical characteristics should be done to understand the statistical correlation, 
if any, between the physical and spectral properties of soils at FHL. It also may be advantageous to determine whether plant type and vegetation coverage at this site show any correlation to soil strength.

Future data collections will incorporate the additional bands included in Digital Globe's WV3 satellite. WV3 launched in April 2014 and senses the same 8 multispectral bands as WV2, plus 8 shortwave infrared (SWIR) bands ranging from 1195 to $2365 \mathrm{~nm}$ and 12 clouds, aerosols, vapors, ice, and snow (CAVIS) bands ranging from 405 to $2245 \mathrm{~nm}$. These CAVIS bands will help to mitigate effects caused by the atmosphere, and the SWIR bands will allow for a closer look at surface materials that present unique reflectance and absorption features (Longbotham et al. 2014). WV3 also has higher spatial resolution than WV2, with multispectral resolution of $1.2 \mathrm{~m}$ ground sample distance, SWIR resolution of $3.7 \mathrm{~m}$, and CAVIS resolution of $30 \mathrm{~m}$ resolution (Longbotham et al. 2014). WV3 is the first commercial satellite imaging system with high spatial resolution and SWIR capabilities (Kruse and Perry 2013). It is also the first commercial system to offer both visible and near infrared (VNIR) and SWIR multispectral capabilities (Kruse and Perry 2013). Transitioning to WV3 would allow for greater spectral and spatial resolution although hyperspectral resolution may be necessary to discern subtle reflectance changes from physical soil properties. Figure 17 shows the bands for WV2 and the subsequent WorldView-3 (WV3) sensors.

Other image analysis techniques should also be explored to improve the accuracy of the results. Spectral unmixing is a technique conventionally used with hyperspectral data to unmix, or separate, the contents of a pixel based on decomposing the measured spectra into a collection of endmembers and their relative abundances. Endmembers represent individual components, such as vegetation or soil that jointly occupy the same pixel (Keshava and Mustard 2002). This technique may be helpful to unmix the effects of vegetation from the spectral signature. 
Figure 17. The difference in band wavelengths between WV2 and WV3 sensors.

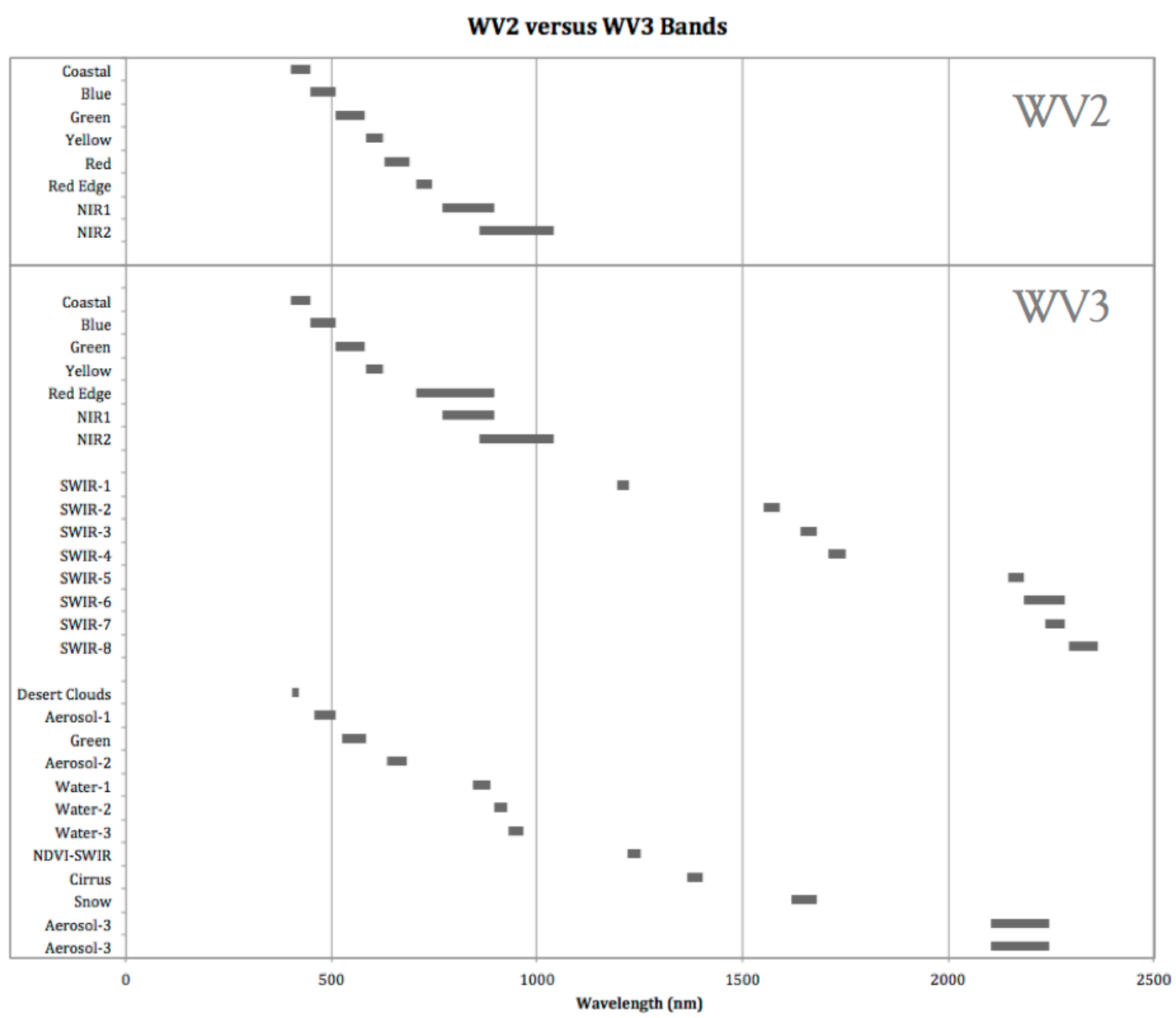

Furthermore, expanded use of training and holdback sites would allow for a more accurate and complete assessment of the results of this process. This would require taking field measurements at more sites of varying strength to have sufficient data to randomly select sites to holdback during the training and classification process. Also, masking out visually discernible unsuitable areas (like alluvial wash) before running the classifications might be helpful. Some known sites have similar spectral properties but very different engineering properties, as described in Section 4.1. Visually inspecting the imagery and removing before analysis those areas that are obviously unsuitable would concentrate the research in the most critical study areas. 


\section{References}

Al-Amoudi, O. S. B., I. M. Asi, Hamad I. A. Wahhab, and Z. A. Khan. 2002. Clegg Hammer-California-Bearing Ratio Correlations. J ournal of Materials in Civil Engineering 14 (6): 512- 523.

ASTM. 2005. Standard Test Method for CBR (California Bearing Ratio) of LaboratoryCompacted Soils. West Conshohocken, PA: ASTM International.

Bachmann, C. M., T. L. Ainsworth, and R. A. Fusina. 2006. Improved Manifold Coordinate Representations of Large-Scale Hyperspectral Scenes. IEEE Transactions on Geoscience and Remote Sensing 44 (10): 2786- 2803.

Bachmann, C. M., M. J . Montes, and R. A. Fusina. 2012. NRL Hyperspectral Imagery Trafficability Tool (HITT): Software and Spectral-Geotechnical Look-up Tables for Estimation and Mapping of Soil Bearing Strength from Hyperspectral Imagery. NRL/MR/ 7230--12-9365. Washington, DC: Naval Research Laboratory, Remote Sensing Division.

Ben-Dor, E., K. Patkin, A. Banin, and A. Karnieli. 2002. Mapping of Several Soil Properties Using DAIS-7915 Hyperspectral Scanner Data-A Case Study over Clayey Soils in Israel. International J ournal of Remote Sensing 23 (6): 10431062.

Ben-Dor, E., N. Goldlshleger, Y. Benyamini, M. R. Agassi, and D. G. Blumberg. 2003. The Spectral Reflectance Properties of Soil Structural Crusts in the 1.2- to 2.5- $\mu \mathrm{m}$ Spectral Region. Soil Science Society of America J ournal 67 (1): 289- 299.

Clegg. 2011. CBR (less than 50) and the Medium (2.25 kg-5 lb.) Clegg Impact Soil Tester. Wembley, Australia: Dr. Baden Clegg Pty Ltd.

Digital Globe. 2016. The Largest Constellation in the Industry. https://www.digitalglobe.com/about-us/content-collection (accessed 28 March 2016.).

Dutta, D., A. E. Goodwell, P. Kumar, J . E. Garvey, R. G. Darmody, D. P. Berretta, and J . A. Greenberg. 2015. On the Feasibility of Characterizing Soil Properties From AVIRIS Data. IEEE Transactions on Geoscience and Remote Sensing 5 (9): 5133- 5147.

Ge, Y., C. L. S. Morgan, S. Grunwald, D. J . Brown, and D. V. Sarkhot. 2011. Comparison of Soil Reflectance Spectra and Calibration Models Obtained Using Multiple Spectrometers. Geoderma 161 (3): 202-211.

Harris Geospatial Solutions. 2016a. Calculate Confusion Matrices. http://www.harrisgeospatial.com/docs/CalculatingConfusionMatrices.html\#UseROI (accessed 29 March 2016).

_. 2016b. Maximum Likelihood. http://www.harrisgeospatial.com/docs/MaximumLikelihood.html (accessed 29 March 2016). 
Hines, C., and M. Wolboldt. 2008. Predicting Soil Type from Remotely Sensed Data. In Proceedings of the Transportation Systems Workshop, April, Phoenix, AZ.

Keshava, N., and J. F. Mustard. 2002. Spectral Unmixing. Signal Processing Magazine, IEEE 19 (1): 44- 57.

Kruse, F. A. 2015. Final Report: Soil Characterization Using Spectral Measurements. NPS/ CRREL FY15 Collaboration. Monterey, CA: Naval Postgraduate School, Remote Sensing Center.

Kruse, F. A., and S. L. Perry. 2013. Mineral Mapping Using Simulated Worldview-3 Short-Wave-Infrared Imagery. Remote Sensing 5 (6): 2688- 2703.

Longbotham, N., F. Pacifici, B. Baugh, and G. Camps-Valls. 2014. Prelaunch Assessment of WorldView-3 Information Content. In IEEE GRSS Workshop on Hyperspectral Image and Signal Processing (WHISPERS), 479- 486.

Middlebrooks, T. A., and G. E. Bertram. 1950. Development of CBR Flexible Pavement Design Method for Airfields: A Symposium: Adaptation to the Design of Airfield Pavements. Transactions of the American Society of Civil Engineers 115 (1): 468-471.

Pass, M., R. Brinson, and L. An. N.d. Improving Intelligence Discovery in Sensor Data and Imagery Using Revelatum ${ }^{\mathrm{TM}}$ Emergence Visualization. Arlington, VA: DAn Solutions.

Richards, J . A. 1999. Remote Sensing Digital Image Analysis. Vol. 3. New York: Springer.

Shaw, G. A., and H. K. Burke. 2003. Spectral Imaging for Remote Sensing. Lincoln Laboratory J ournal 14 (1): 3- 28.

Shepherd, K. D., and M. G. Walsh. 2002. Development of Reflectance Spectral Libraries for Characterization of Soil Properties. Soil Science Society of America J ournal 66 (3): 988- 998.

Sim, J ., and C. C. Wright. 2005. The Kappa Statistic in Reliability Studies: Use, Interpretation, and Sample Size Requirements. Physical Therapy 85 (3): 257268.

Sørensen, L. K., and S. Dalsgaard. 2005. Determination of Clay and Other Soil Properties by Near Infrared Spectroscopy. Soil Science Society of America J ournal 69 (1): 159- 167.

Viscarra Rossel, R. A., D. J . J . Walvoort, A. B. McBratney, L. J . J anik, and J . O. Skjemstad. 2006. Visible, Near Infrared, Mid Infrared or Combined Diffuse Reflectance Spectroscopy for Simultaneous Assessment of Various Soil Properties. Geoderma 131 (1): 59- 75. 


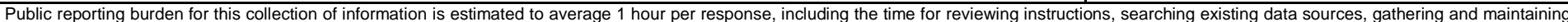

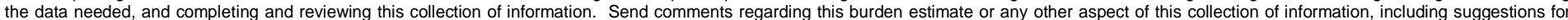

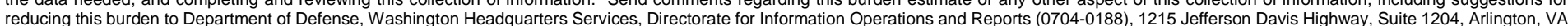

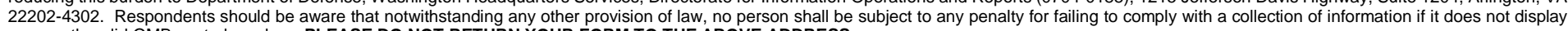
a currently valid OMB control number. PLEASE DO NOT RETURN YOUR FORM TO THE ABOVE ADDRESS.
1. REPORT DATE (DD-MM-YYYY)
2. REPORT TYPE
August 2016
Technical Report/Final

3. DATES COVERED (From - To)

\section{TITLE AND SUBTITLE}

Image Analysis and Classification Based on Soil Strength

5a. CONTRACT NUMBER

5b. GRANT NUMBER

5c. PROGRAM ELEMENT NUMBER

\section{AUTHOR(S)}

Ariana M. Sopher, Sally A. Shoop, Jesse M. Stanley, Jr., and Brian T. Tracy

\section{5d. PROJECT NUMBER}

5e. TASK NUMBER

5f. WORK UNIT NUMBER

6CJ4D3

\section{PERFORMING ORGANIZATION NAME(S) AND ADDRESS(ES)}

8. PERFORMING ORGANIZATION REPORT NUMBER

U.S. Army Engineer Research and Development Center (ERDC)

Cold Regions Research and Engineering Laboratory (CRREL)

ERDC/CRREL TR-16-13

72 Lyme Road

Hanover, NH 03755-1290

\section{SPONSORING I MONITORING AGENCY NAME(S) AND ADDRESS(ES)}

Army Terrestrial Environmental Modeling and Intelligence System (ARTEMIS)

U.S. Army Engineer Research and Development Center (ERDC)

Cold Regions Research and Engineering Laboratory (CRREL)

72 Lyme Road

Hanover, NH 03755-1290

\section{DISTRIBUTION / AVAILABILITY STATEMENT}

Approved for public release; distribution is unlimited.

\section{SUPPLEMENTARY NOTES}

Geospatial Remote Assessment for Ingress Locations (GRAIL) Project

\section{ABSTRACT}

Satellite imagery classification is useful for a variety of commonly used applications, such as land use classification, agriculture, wetland delineation, forestry, geology, and landslide potential. However, image classification for physical properties of surface soils, such as strength or bearing capacity, is often obscured by other surface conditions, such as moisture and vegetation, although these are also indicators of soil strength. This project used remote methods of terrain analysis to search for areas suitable for vehicle or aircraft maneuverability based on slope, roughness, vegetation, soil type, and wetness and also performed direct classification of imagery based on soil strength. Using a maximum likelihood supervised classification approach, trained by a limited amount of ground-truth strength measurements, a soil strength classification was applied to WorldView-2 multispectral satellite imagery. This paper presents the work done on the imagery classification for soil strength, the apparent relationship between the reflectance and soil strength, and the ongoing work to expand the technique to new imagery by using existing training sets.

\section{SUBJECT TERMS}

Digital image classification, Mobility, Multispectral imaging, Remote-sensing images, Shear strength of soils, Soil moisture-Measurement, Soils--Testing, Soil trafficability

\section{SECURITY CLASSIFICATION OF:}

\section{a. REPORT}

Unclassified

\section{b. ABSTRACT}

Unclassified

\section{c. THIS PAGE}

Unclassified

\begin{tabular}{c|c|} 
17. LIMITATION & $\begin{array}{c}\text { 18. NUMBER } \\
\text { OF ABSTRACT }\end{array}$ \\
OF PAGES \\
SAR & 32 \\
\end{tabular}

19a. NAME OF RESPONSIBLE PERSON

19b. TELEPHONE NUMBER (include area code) 